\title{
Habitat Design Considerations for Implementing Solar Particle Event Radiation Protection
}

\author{
Matthew A. Simon and Martha Clowdsley \\ NASA Langley Research Center, Hampton, VA, 23681, USA \\ Steven Walker \\ Old Dominion University, Norfolk, VA 23529, USA
}

\begin{abstract}
Radiation protection is an important habitat design consideration for human exploration missions beyond Low Earth Orbit. Fortunately, radiation shelter concepts can effectively reduce astronaut exposure for the relatively low proton energies of solar particle events, enabling moderate duration missions of several months before astronaut exposure (galactic cosmic ray and solar particle event) approaches radiation exposure limits. In order to minimize habitat mass for increasingly challenging missions, design of radiation shelters must minimize dedicated, single-purpose shielding mass by leveraging the design and placement of habitat subsystems, accommodations, and consumables. NASA's Advanced Exploration Systems RadWorks Storm Shelter Team has recently designed and performed radiation analysis on several low dedicated mass shelter concepts for a year-long mission. This paper describes habitat design considerations identified during the radiation analysis phase of the study. These considerations include placement of the shelter within a habitat for improved protection, integration of human factors guidance for sizing shelters, identification of potential opportunities for habitat subsystems to compromise on individual subsystem performances for overall vehicle mass reductions, and pre-configuration of shelter components for reduced deployment times.
\end{abstract}

\section{Nomenclature}

Acronyms

$\begin{array}{ll}\text { AES } & =\text { Advanced Exploration Systems } \\ B E O & =\text { Beyond Earth Orbit } \\ B N N T & =\text { Boron Nitride Nanotubes } \\ C A D & =\text { Computer-Aided Design } \\ C T B & =\text { Cargo Transfer Bag } \\ D S H & =\text { Deep Space Habitat } \\ G C R & =\text { Galactic Cosmic Rays } \\ H A T & =\text { Human Spaceflight Architecture Team } \\ H D U & =\text { Habitat Demonstration Unit }\end{array}$

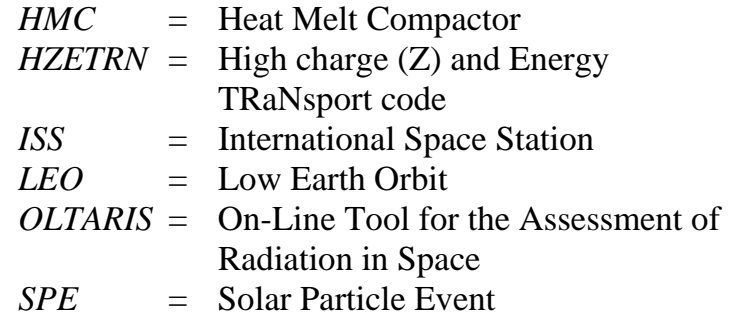

\section{Introduction}

T XPOSURE of astronaut crews to the deep space radiation environments increase the risk of deleterious Ehysiological effects such as radiation sickness and late-term effects, central nervous system damage, and increased incidence of debilitating or fatal cancers. Mitigating these radiation-related health risks in an affordable and sustainable manner is a major challenge in designing for human exploration of the Solar System beyond Earth orbit (BEO). Since pharmaceutical treatments and astronaut screening based upon genetic radiation resistance have not yet been proven to provide sufficient protection, shielding is the primary method available to mitigate radiation exposure. Shielding in the form of polyethylene tiles has been implemented successfully on the International Space Station (ISS); enabling longer stays and demonstrating adequate protection can be achieved in Low Earth Orbit (LEO). However, as humans travel beyond the protection of the Earth's magnetic field, the radiation environment becomes significantly more challenging and requires increased amounts of shielding to reduce astronaut exposure and mitigate radiation-related risks. 
The deep space radiation environment beyond the Earth's magnetosphere results from the combination of galactic cosmic rays (GCR), solar particle events (SPEs), and secondary particles created by interactions between the charged ions of these environments and shielding materials surrounding the astronauts. GCR consist of charged particles which exist pervasively throughout the galaxy. Some GCR particles are difficult to shield with practical amounts of shielding because of their high energies and the secondary particles which can be formed by their collision with shielding materials. The rates of exposure associated with GCR are relatively low, but also extremely difficult to mitigate without large amounts of shielding (on the order of a few meters). This limits acceptable mission durations until innovative solutions to the GCR problem are developed. In contrast to GCR, large SPEs are rare, short-lived (durations are measured in hours), high-exposure rate events. Fortunately, shielding materials (particularly those with high concentrations of hydrogen) are effective at reducing astronaut exposure to these events because SPEs are made up primarily of low and medium energy protons. Therefore, if adequate shielding is provided and astronauts receive sufficient warning to enter the sheltered area, the SPE risk can be managed through appropriate vehicle design considerations.

Adequate shielding could be provided by simply surrounding a vehicle with a large enough mass. However, it is cost prohibitive to launch the mass necessary to shield an entire space habitat. It is also undesirable to carry single-purpose shielding mass dedicated only to provide radiation shielding (i.e. parasitic mass) when logistics and subsystems necessary to support humans can provide sufficient shielding. To address this design problem, the RadWorks Storm Shelter team was created and tasked with designing low parasitic mass SPE shelter concepts which leverage available logistics and subsystems to shield smaller areas within a habitat. In performing radiation analyses of the shelter concepts within this notional vehicle layout, many habitat and shelter design considerations were identified which could reduce the overall vehicle mass and provide better protection to crews.

In order to enable human exploration missions beyond the Earth's magnetic field, habitat masses must be optimized through an integrated design approach which concurrently considers radiation shielding, subsystem design, habitat interior layout design, and crew operations. This paper first describes the RadWorks Storm shelter study from FY`12 including descriptions of the shelter concepts investigated and the habitat design assumed for the study in Section II. Section III describes design guidance which has been observed from RadWorks shelter designs and radiation analysis results including: the positions of interior logistics, the size and location of the protected area, material choices, and design compromises for improved habitability. Finally, recommendations are characterized by their potential impact to system mass and improved habitability.

\section{RadWorks SPE Shelter Design Effort}

NASA's Advanced Explorations Systems (AES) projects were created to advance the readiness levels of existing technologies to the level needed for exploration missions. The AES RadWorks Storm Shelter team focuses on reducing astronaut risk to space radiation exposure through the development of low parasitic mass SPE shelter concepts. The RadWorks Storm Shelter team was charged with creating designs applicable to a one year mission with four crew in the deep space radiation environment. Each of these shelters was designed to be deployable by two crew in less than an hour and capable of housing astronauts for up to 36 hours. A large design space of shelter variants was narrowed to the four concepts shown in Figure 1:1) a deployable group shelter based upon existing internal structure, 2) a wearable, sleeping-bag derived shielding system, 3) an individual crew quarters based solution, and 4) a deployable individual shelter. 
1.

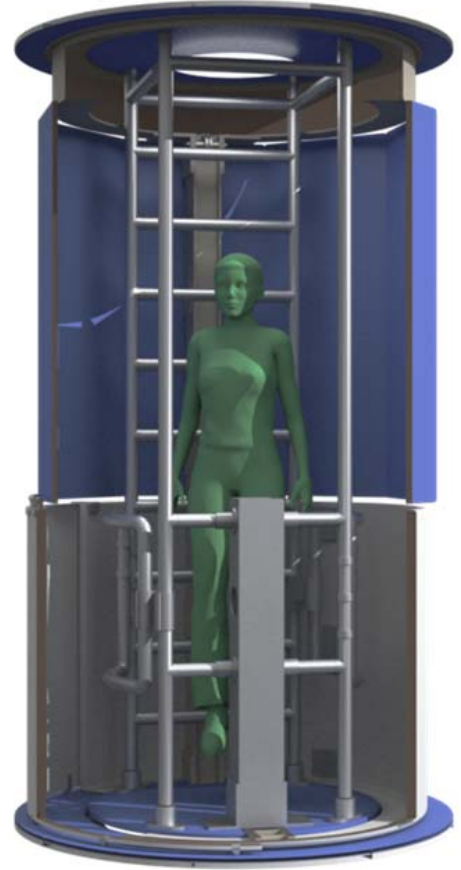

2.

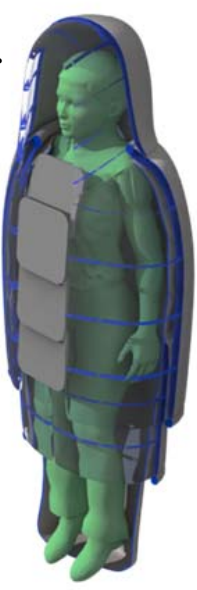

3.

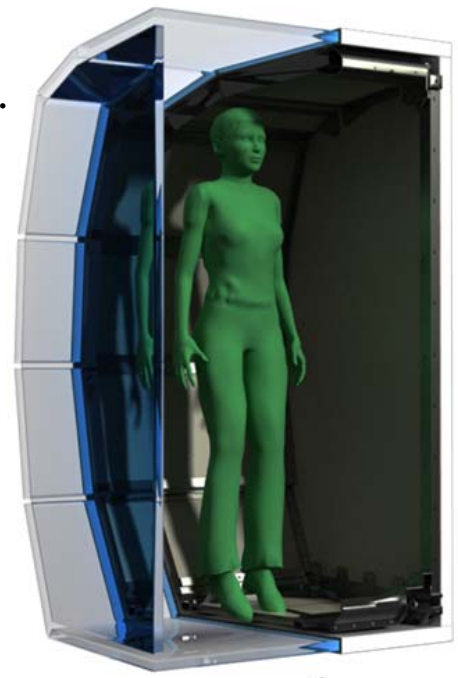

4.

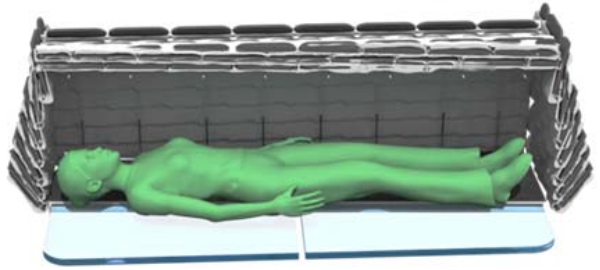

Figure 1: Four storm shelter concepts analyzed in FY`12 study

1) The deployable group shelter features repurposing of structural floor and ceiling panels to provide a group shelter in the center of the habitat. These panels are made of a composite sandwich honeycomb structure which could be filled with water or used as a structural framework for the attachment of logistics.

2) The wearable, sleeping bag derived shelters are constructed from water bladders and logistics to shield individual astronauts in their crew quarters without disrupting crew operations substantially. They also allow some degree of crew mobility during an event. They are also potentially useful for other vehicles designed for shorter duration missions.

3) Individual crew quarters based shelters utilize crew quarters similar to those in the International Space Station, but with integrated hollow structural walls that would be filled with contingency life support water to provide radiation protection. These could either be prefilled or filled upon need when a SPE is detected. The shelter inner mold line was preserved by expanding the crew quarters outer mold line. The interior volume of crew quarters has been proven on ISS for long durations of occupation (at least 6 months).

4) The deployable individual shelter concept features a base which serves as a two-part hinged contingency water containment system and a set of U-shaped rods that serve as a structural frame for the attachment of logistics. These logistics could be prearranged into easy to deploy sheets which would them be draped over the frame to shield individual astronauts.

Designs for each of these four concepts were evaluated based upon a primary figure of merit: the parasitic mass required to achieve the required radiation dose reduction. Calculating parasitic mass requires a detailed radiation analysis to determine the amount of protection material necessary to reduce effective dose, which can then be compared with available materials from the habitat to determine how much of the shielding mass needs to be parasitic. Detailed radiation analyses were performed on each of the four concepts ${ }^{1}$. Each radiation analysis consisted of two parts: a geometry preparation/handling step and a transport code ray-tracing analysis step. The habitat selected for the FY`12 study was a deep space adaptation of the Habitat Demonstration Unit (HDU) ${ }^{2}$. This demonstration platform was developed at NASA Johnson Space Center for testing and evaluation of architectural configurations and mission operations. It was chosen based upon its possible use as a demonstration platform for full-scale demonstrations of shelter concepts. It is composed of three modules shown in Figure 2. The main module features three levels: the main floor housing many of the work functions; the lower loft (second floor) housing crew quarters, living areas, and logistics; and the upper loft which serves as an attic for logistics storage. An airlock and a hygiene module were attached to the radial ports of the HDU. These are analogous to an in-space airlock and a logistics module for the deep space mission. The HDU Computer-Aided Design (CAD) model was outfitted with 
geometries representing the logistics and subsystems necessary for a 1 year deep space mission assuming no resupply. This outfitting was parametrically sized using tools and assumptions consistent with the conceptual design of the Deep Space Habitat (DSH) for NASA's Human Spaceflight Architecture Team (HAT) ${ }^{3}$. The structure, subsystems, and logistics were then assigned densities based upon the parametric sizing and previous studies ${ }^{4-7}$. Finally, human models were placed within the interior to identify the dosimetry points necessary to calculate the equivalent exposure of astronauts.
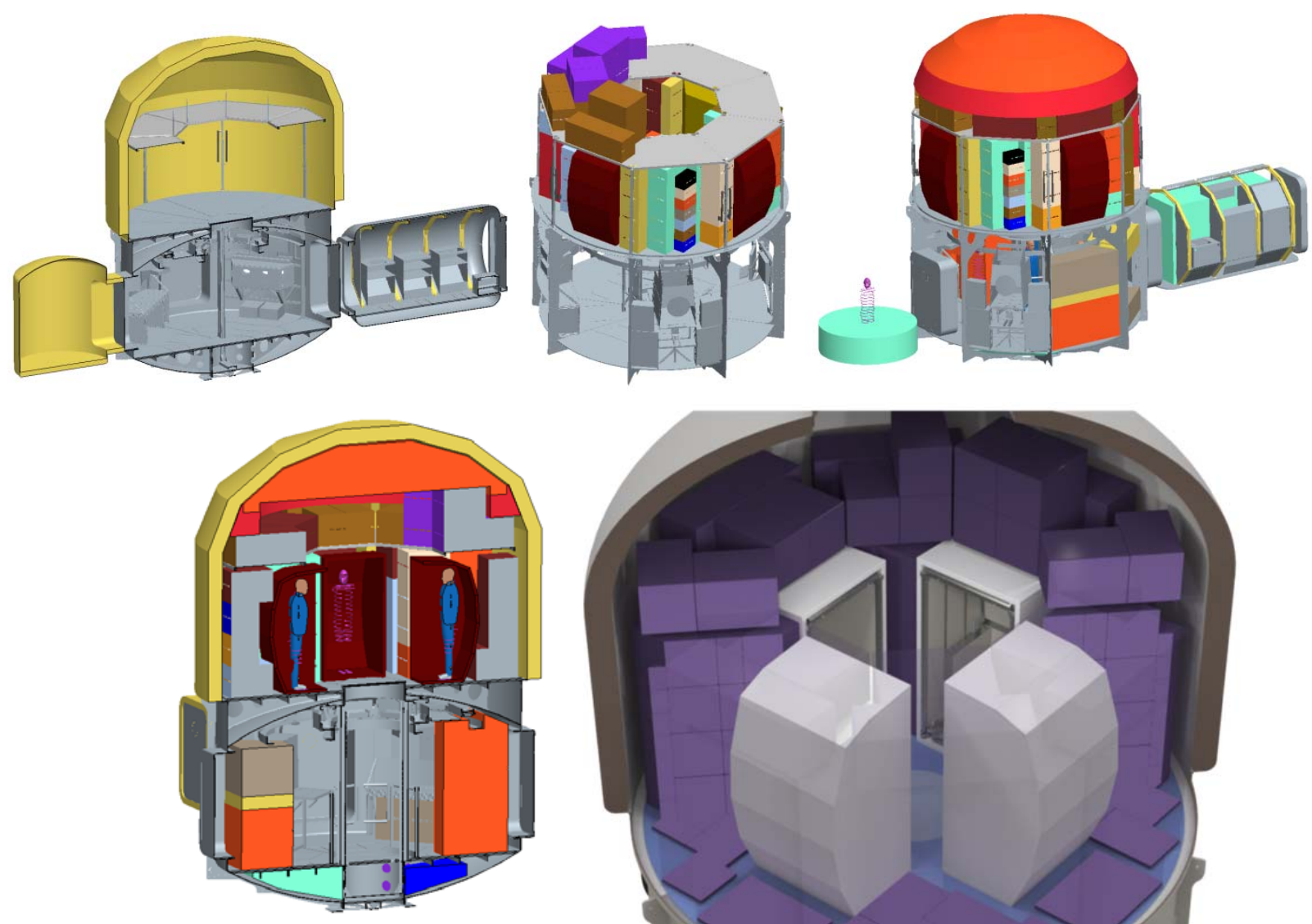

Figure 2: Outfitting and layout of the Habitat Demonstration Unit for one year mission

The first step in the radiation analysis was to utilize scripts to generate ray traces of the CAD habitat geometry and human models. These ray traces calculate how much material is between the target point and the deep space radiation environment in all directions. An example of a ray trace performed for ISS is shown in Figure 3. The scripts and codes contained in the On-Line Tool for the Assessment of Radiation in Space (OLTARIS) ${ }^{8}$ were used to combine vehicle ray traces with human body ray traces for a large number of locations in the human body, calculate the transport of the external environment along each ray to each body point, and utilize these results to predict astronaut effective dose ${ }^{9}$ for the chosen external environment. OLTARIS uses the High charge $(\mathrm{Z})$ and Energy TRaNsport code (HZETRN) ${ }^{10,11}$ (developed at NASA Langley) as the transport algorithm. The body model chosen was the Female Adult voXel model (FAX) ${ }^{12}$. A probabilistic SPE spectrum, based on a model developed by Xapsos $^{13}$ and representing a 95\% confidence level for total SPE exposure for a one year mission, was used as a design basis environment for these calculations. In addition, several different SPE models were investigated to understand the impact of different magnitude and spectral shaped events on the required shielding thicknesses. An in-depth description of this process and the assumptions used in this analysis are covered in more detail in Ref. 1. 


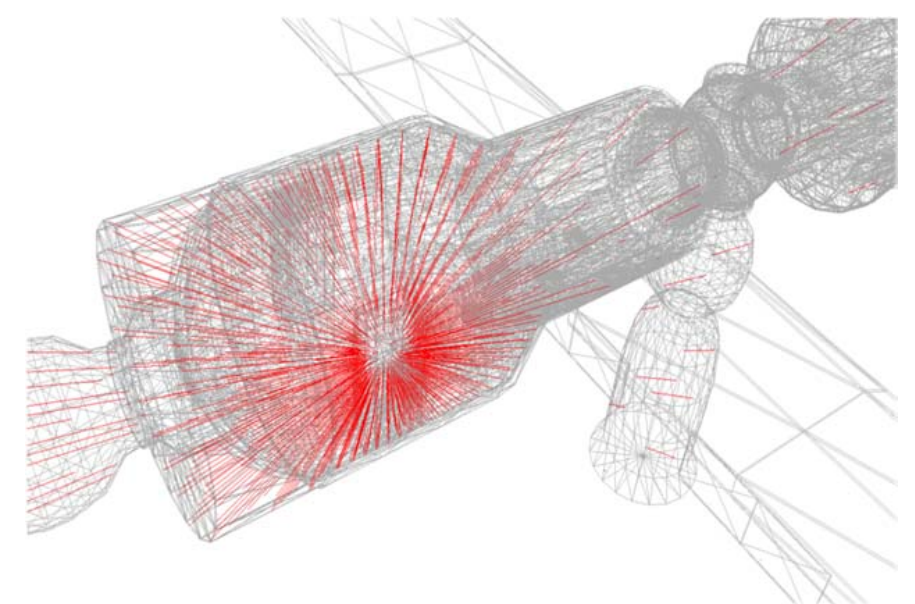

Figure 3: Example ray trace using the International Space Station. The red line segments are the intersection of rays with the ISS. Figure courtesy of Garry Qualls, NASA Langley Research Center.

Theradiation analysis was performed on the outfitted deep space HDU in two phases. The first phase calculated astronaut exposure in the habitat in its normal configuration without any shelter concepts deployed to set baseline exposure values against which the shelter concept's effectiveness could be evaluated. This calculation was performed for two different astronaut locations: one in the crew quarters and one in the main section central area. In the second phase, the astronaut exposures within each deployed shelter concept were evaluated at multiple locations in the habitat. These values were compared to the baseline non-shielded exposures in terms of the percent reduction in astronaut exposure to estimate shelter effectiveness. Shielding concepts met the required exposure reduction if, for the Xapsos 95\% event, the effective dose for the astronaut inside the concept was less than $50 \%$ of the baseline value for the habitat in its normal configuration. However, the goal was a reduction of $70 \%$. Effective dose values for shielding concepts in the crew quarters were compared to the baseline value of $450 \mathrm{mSv}$ and effective dose values for shielding concepts in the main section were compared to the baseline value of $361 \mathrm{mSv}$. Table 1-4 show the results of the radiation analyses (shielding thicknesses and total shielding masses) for each of the shelter concepts at various deployment locations in the habitat and for various analysis assumptions. Ranges in the thickness data indicate the ranges of thicknesses required for either multiple individual shelter units simultaneously deployed or multiple locations of crew within a group shelter.

Table 1: Radiation analysis results for deployable group shelter leveraging available structure

\begin{tabular}{|c|c|c|c|c|}
\hline & \multicolumn{2}{|c|}{ Water Wall Thickness, in } & \multicolumn{2}{c|}{ Total Mass of Water, lbm } \\
\cline { 2 - 5 } & $\mathbf{5 0 \%}$ & $\mathbf{7 0 \%}$ & $\mathbf{5 0 \%}$ & $\mathbf{7 0 \%}$ \\
\hline Panels Only & $1.04-1.65$ & $4.04-5.00$ & 1696 & 5785 \\
\hline $\begin{array}{c}\text { Panels Plus } \\
\text { Logistics }\end{array}$ & 0 & $1.67-2.85$ & 0 & 2677 \\
\hline
\end{tabular}

Table 2: Radiation analysis results for wearable, sleeping bag derived shelters

\begin{tabular}{|c|c|c|c|c|}
\hline \multirow{2}{*}{} & \multicolumn{2}{|c|}{ Water Wall Thickness, in } & \multicolumn{2}{c|}{$\begin{array}{c}\text { Total Mass for 4 } \\
\text { Astronauts, lbm }\end{array}$} \\
\cline { 2 - 5 } & $\mathbf{5 0 \%}$ & $\mathbf{7 0 \%}$ & $\mathbf{5 0 \%}$ & $\mathbf{7 0 \%}$ \\
\hline $\begin{array}{c}\text { Wearable Shield in } \\
\text { Crew Quarters }\end{array}$ & 2.1 & 5.0 & 1527 & 3636 \\
\hline $\begin{array}{c}\text { Wearable Shield in } \\
\text { Main Section }\end{array}$ & 2.8 & 6.1 & 2036 & 4436 \\
\hline
\end{tabular}

Table 3: Radiation analysis results for individual crew quarters based shelters

\begin{tabular}{|l|c|c|c|c|}
\hline \multirow{2}{*}{} & \multicolumn{2}{|c|}{ Food/Brick Layer Thickness, in } & \multicolumn{2}{c|}{$\begin{array}{c}\text { Total Mass for 4 } \\
\text { Astronauts, lbm }\end{array}$} \\
\cline { 2 - 5 } & $\mathbf{5 0 \%}$ & $\mathbf{7 0 \%}$ & $\mathbf{5 0 \%}$ & $\mathbf{7 0 \%}$ \\
\hline Modeled as Water & $0.52-0.92$ & $3.43-4.42$ & 627 & 3693 \\
\hline
\end{tabular}

5

American Institute of Aeronautics and Astronautics 


\begin{tabular}{|c|c|c|c|c|}
\hline $\begin{array}{c}\text { Modeled as } \\
\text { Aluminum }\end{array}$ & $0.27-0.50$ & $2.14-3.01$ & 905 & 6520 \\
\hline
\end{tabular}

Table 4: Radiation analysis results for deployable individual shelter

\begin{tabular}{|c|c|c|c|c|}
\hline \multirow{2}{*}{} & \multicolumn{2}{|c|}{ Water Wall Thickness, in } & \multicolumn{2}{c|}{ Total Mass of Water, } \\
\cline { 2 - 5 } & $\mathbf{5 0 \%}$ & $\mathbf{7 0 \%}$ & $\mathbf{5 0 \%}$ & $\mathbf{7 0 \%}$ \\
\hline $\begin{array}{c}\text { Crew Quarters in } \\
\text { Original Position }\end{array}$ & 2.7 & 7.7 & 3119 & 8942 \\
\hline $\begin{array}{c}\text { Crew Quarters } \\
\text { Moved Inward }\end{array}$ & $0.49-0.84$ & $4.95-5.12$ & 676 & 5827 \\
\hline $\begin{array}{c}\text { Astronauts Doubling } \\
\text { Up }\end{array}$ & $0-0.82$ & $3.50-4.93$ & 379 & 2656 \\
\hline
\end{tabular}

Three major conclusions can be drawn from this data. First, achieving $70 \%$ effective dose reduction for the Xapsos 95\% event is much more challenging than achieving 50\% reduction, resulting in much larger thicknesses. Additionally, it was observed that other SPE models were significantly less demanding than the Xapsos model; those results are not presented here. Second, the thicknesses of shielding materials are highly dependent on the location of the shelter within the habitat and on the habitat design. This is clearly seen in the crew quarters protection concept, where moving the shelter inward reduced the necessary thickness by $~ 35 \%$ for the $70 \%$ effective dose reduction and $\sim 80 \%$ for the $50 \%$ effective dose reduction. Finally, the last major conclusion is that the thicknesses required for the shielding concepts represent non-trivial amounts of mass, which must be reduced through careful integrated design of the habitat concepts.

In addition to the parasitic mass of the concepts determined from the radiation analyses, secondary figures of merit were considered in the design of individual shelter concepts and in the comparison the final designs. These figures of merit were qualitatively assessed to provide feedback to designers on potential issues and to make final selections between concepts for prospective missions. Factors considered include deployment time, a qualitative evaluation of habitability, an assessment of the ease of egress from a shelter during a SPE, and the number of people necessary for nominal deployment operations. Based upon the results from the primary and secondary figures of merit, several recommendations for habitat design have been identified to improve the effectiveness or integration of these types of shelters into a habitat design. The remainder of this paper discusses these recommendations and uses the data from RadWorks Shelter project to provide anecdotal evidence for their value.

\section{Habitat Design Considerations}

Habitat design is a complex multidisciplinary problem where design decisions have significant impacts across subsystems and vehicles. Integration of radiation protection into habitat designs is enhanced by an understanding of how these design decisions affect the effective dose for a given mission. The remainder of this paper is devoted to describing the habitat design considerations for more effective integration of SPE radiation protection hardware and recommendations for overall habitat mass reduction which were observed from the radiation analysis data and its discussion.

\section{A. Size and Shape of Habitat}

The radiation analysis of the HDU CAD model showed that effective dose reduction is best achieved by uniform omnidirectional shielding provided the thickness in each direction is sufficient to mitigate the energy of incoming particles. During the radiation analysis, protected areas uniformly shielded with moderate amounts of shielding materials yielded lower effective doses than slightly heavier shielded areas with gaps or thin spots. The size and shape of the habitat pressure shells have advantages and disadvantages for implementing radiation protection strategies based upon how well they facilitate this uniformity. Assuming a fixed mission (duration, number of crew, amount of required equipment), larger habitats are often chosen because they offer more space for improved habitability. Larger volumes also increase flexibility of maneuvering interior subsystems, logistics, and the defined radiation protection area to achieve adequate protection. The disadvantages of growing habitat size are two-fold. First, the mass of the pressure shell and subsystems to support larger volumes increases proportionally to the volume and surface area of the habitat. Second, the potential for geometric gaps between accommodations and logistics can increase with some interior layout strategies, particularly layouts which arrange interior items along increasingly large outer walls. This is illustrated in Figure 4. This gap issue is readily mitigable by maintaining a small uniform 
arrangement of interior items (subsystems, logistics, etc.) around a similarly sized radiation protection area independent of habitat size. The concern with this strategy is that it limits the potential habitability gains achieved by spreading out interior items.

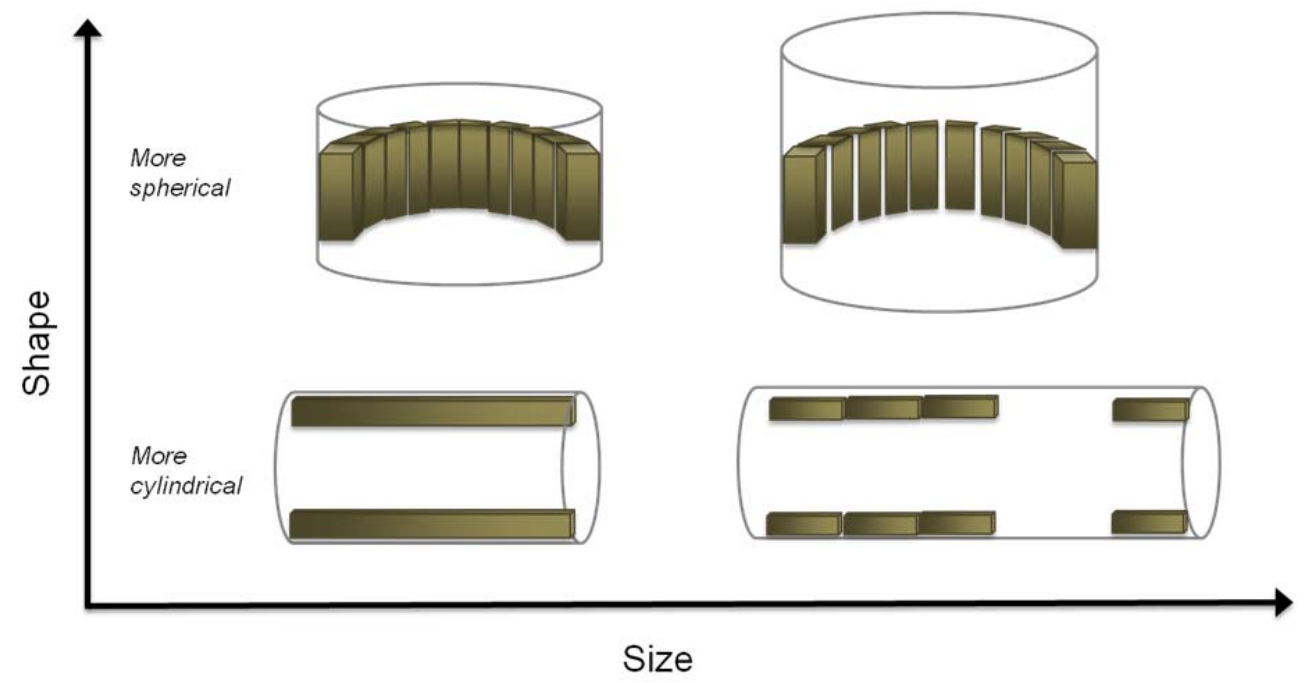

Figure 4: Impact of habitat size and shape on radiation protection

For a fixed volume, habitat shape can also affect a habitat design's radiation protection strategy, as shown in Figure 4. Holding the internal volume constant, a habitat approaching a spherical shape (similar length and diameter) offers increased flexibility to provide a thicker layer of uniform protection. A long cylindrical habitat cannot provide the same amount of uniform protection in the radial direction, but if the layer of shielding materials is sufficient in those directions then the additional protection offered by the larger diameter habitats would be beneficial, but not required.

Finally, habitats tend to be delivered with large propulsion stages, logistics modules, and an Earth-entry capsule for long duration missions. These relatively massive modules can provide significant protection by occluding the habitat from incoming rays. Consideration of the shape/size of the habitat and the location of hatches can reduce necessary shielding mass and increase protection area volume by leveraging placement of the protection area as shown in Figure 5. The radiation shelter tests in the main section could have been improved if the propulsive stage had been modeled or if the shelter were placed in a more occluded zone.
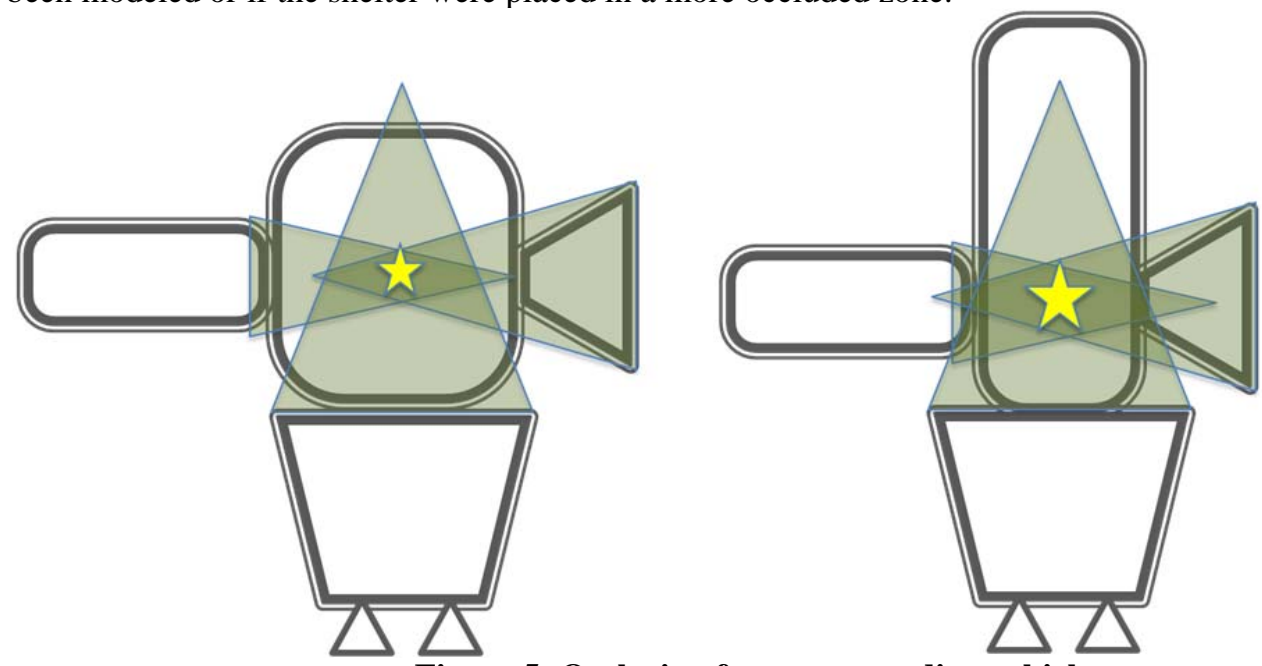

Figure 5: Occlusion from surrounding vehicles

\section{B. Placement of Interior Subsystems, Accommodations, and Logistics}

American Institute of Aeronautics and Astronautics 
Strategic placement of interior subsystems, accommodations, and logistics is another fundamental strategy discussed for creating uniform shielding coverage and minimizing the parasitic mass of shelter concepts. However, this is also one of the fundamental strategies for improving habitability or work efficiency, and desired placements for improved habitability often come in conflict with desired placements for improved radiation protection. As mentioned in Section III.A and shown in Figure 6, habitability concerns tend to spread subsystems out to increase contiguous volume and prevent interferences between astronauts interacting with hardware, whereas radiation protection favors close groupings of hardware around a protection area to reduce mass and relocation of logistics to construct shelter concepts. Compromise between habitability and radiation protection goals is needed to reduce radiation protection masses without increasing stress on crew or compromising safety.

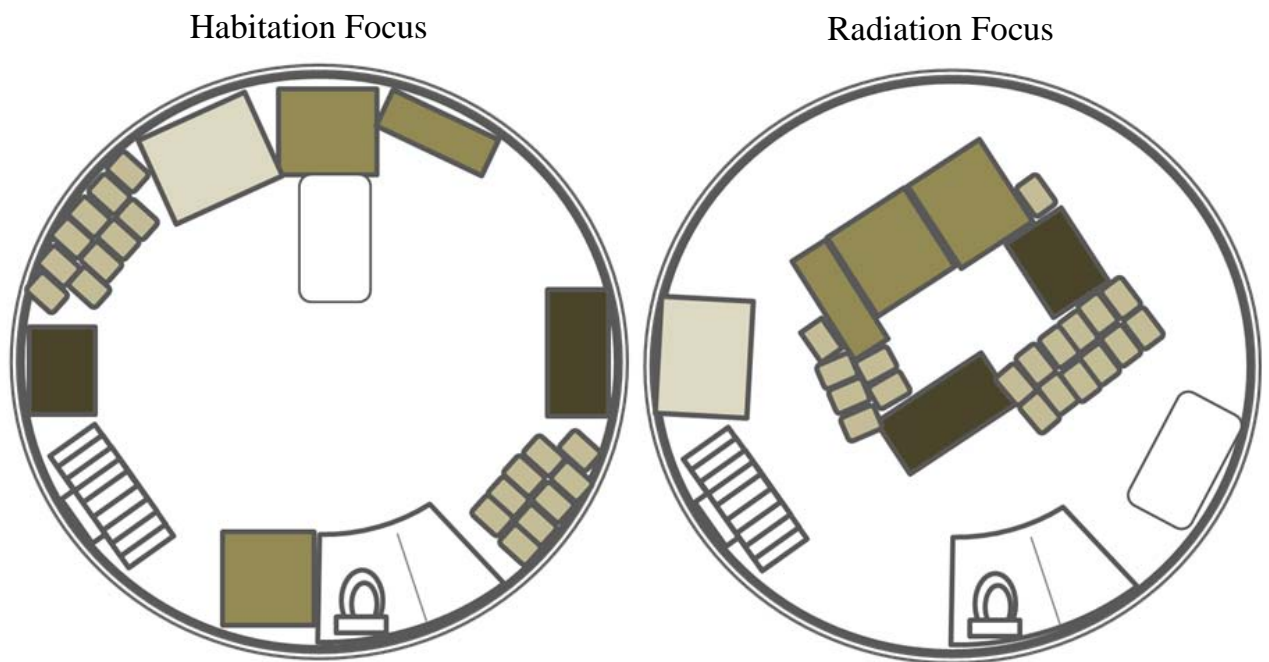

Figure 6: Interior layout designs for habitation and radiation focuses

Several considerations guiding strategic placement of interior items have been identified. First, omnidirectional, uniform coverage requires placement of interior items to ensure adequate material thicknesses in every direction. In addition, increasing the amount of protection around the shelter reduces the mass of the shielding material required (water, logistics, polyethylene, etc.). In the case of water and other consumables, any reduction of shielding materials can also reduce parasitic mass by avoiding a situation in which more of the consumable is necessary for shielding than is nominally being delivered for the mission. The benefit of these approaches can be seen in Table 4, where moving crew quarters inward and filling the vacated space had a significant impact on the parasitic mass and the required waterwall thickness. Another consideration for the arrangement of interior items which will be considered in future RadWorks analyses is the selection of materials surrounding the protection area based upon their ability to mitigate incoming radiation. High densities and high-hydrogen content interior items (e.g., life support water subsystems, other life support systems, food storage, and contingency consumable storage) are prime candidates for colocation with the protection area. These are represented in the notional layout in Figure 6 by darker shades of color. Fortunately, colocation of these high-density, high hydrogen content subsystems and accommodations is either favorable or neutral from a habitability perspective ${ }^{14,15}$.

During shelter concept design meetings, it was also noted that the relocatability of items with high radiation mitigation potentials should also be considered in determining the position of items relative to the protection location. Difficult to move, fixed location subsystems such as life support water processing equipment can only be leveraged effectively if colocated with the protection area. Conversely, in order to increase the local thickness of materials grouped around the protection area during an event, the nominal storage locations of easily relocatable items like food and spares should be separated from the protection area to an area where their relocation will not create gaps around the shelter concept. This is illustrated in Figure 7 and was demonstrated in shelter concept 1. Additionally, the positions of these subsystems can be traded to reduce the amounts of relocatable materials; decreasing deployment time. 


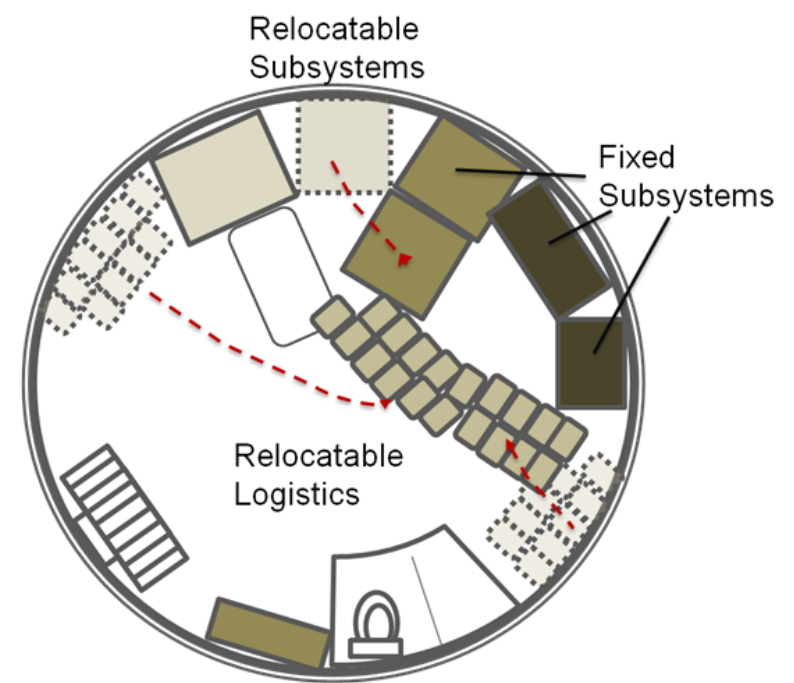

Figure 7: Location positions of relocatable and fixed hardware

\section{Location of Sheltered Area}

As illustrated in Table 2 and Table 4, the absolute positions of shelters within the habitat and relative positions with respect to the interior accommodations are important design consideration with significant impacts on the reduction of effective dose. In particular, centralized placement of the sheltered area resulted in lower exposures (both radially and axially unless utilizing adjacency to large external vehicle for dose reduction). Also, placement of a shelter in the midst of high density, high hydrogen content materials reduced effective doses. In addition, the positions of docking ports, hatches, and windows have an impact upon the location of a shelter. In general, hatches and windows must be kept clear for emergency egress. This can lead to thin protection areas which should be avoided.

The operational functionality and habitability considerations also had an impact on shelter location. In general, group shelters were deployed in translation paths based upon their centralized locations and larger volumes capable of accommodating a crew of 4 . They are also placed relative to important vehicle command and control hardware to maintain situational awareness of the vehicle throughout the event, though it was suggested that miniaturization and consolidation of command and control capabilities to a laptop or mobile device may alleviate this requirements. It was also suggested that the galley/wardroom was a good potential location for the shelter because of the access to goods and a comfortable established group space. Individual shelters are often placed in crew quarters because of the acclimatization of crew to those volumes, the possibility of remote command and control from crew quarters computers, and the ability to either work or sleep during the event. One other consideration proved during the radiation analysis was the location of other individual shelters to leverage adjacent crew members for improved shielding. The nominal locations of the sheltered areas assumed in the study are shown green in Figure 8 (though only 1 of 4 shelters is shown in the upper right deployable individual shelter concept). All of the considerations for placement of the sheltered area will be leveraged in future iterations of the RadWorks Project. 


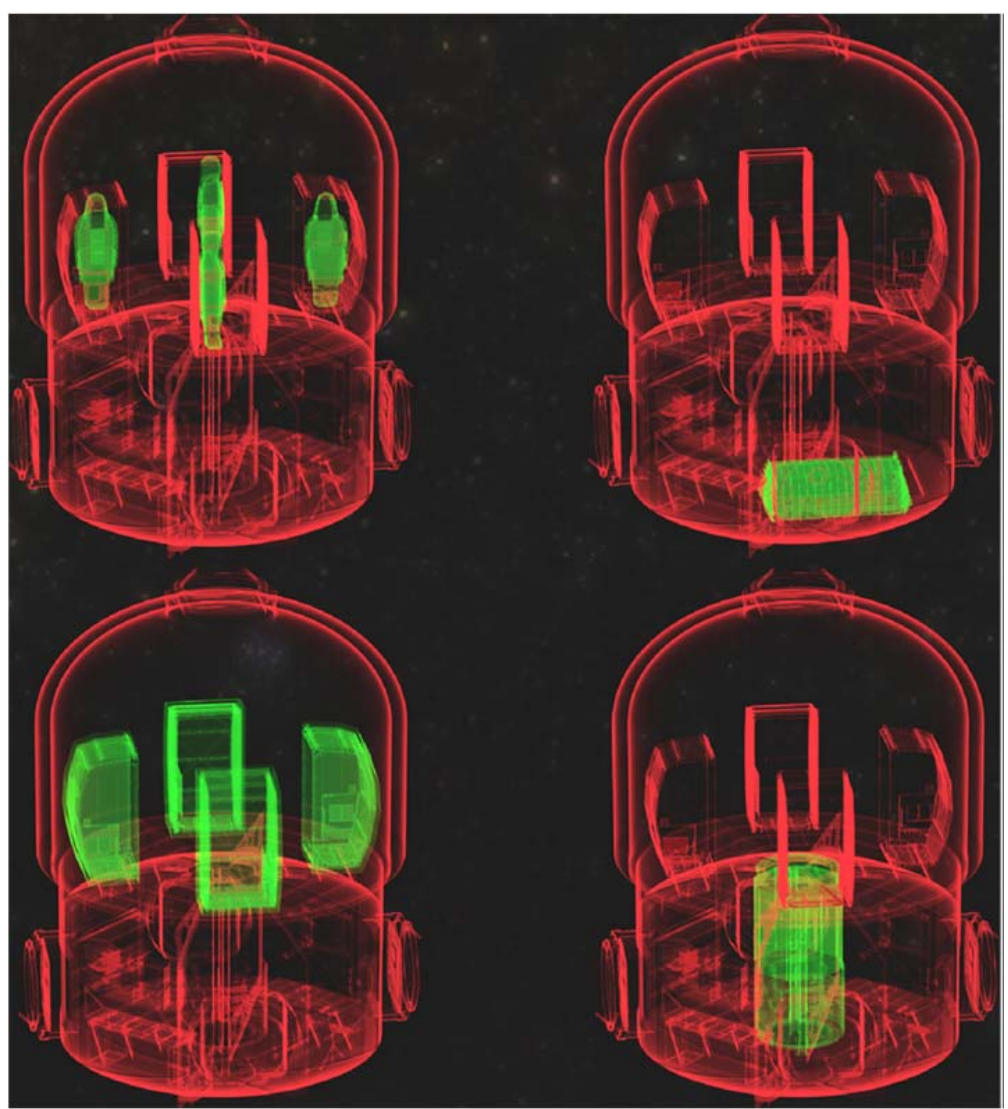

Figure 8: Location and shapes of protected areas

\section{Size and Shape of Protected Area}

The size and shape of the protected area represents a significant trade which was addressed in the design of shelter concepts. A major decision was whether to implement individual shelters or group shelters. Individual shelters can be implemented in existing private areas with modification of existing hardware within the habitat to save mass and improve habitability (e.g., crew quarters). The size and shape of individual shelters is limited by habitability and ventilation requirements. Group shelters can be implemented in a smaller aggregate volume to reduce the surface area of the shelter shielding material. This can be seen by comparison of the group protection shelter in the lower right of Figure 8 (which is designed for 2 crew as shown) to the other concepts in the figure.

In extremely mass constrained situations, the volumes of the shelters used in this study could be reduced to minimum acceptability thresholds, which can be found in coal mining, oil rig, tornado, and bomb shelter documentation and in prison designs for isolated confinement. However, for long duration habitats there is no need for utilizing the minimum acceptable thresholds if logistics are utilized as shielding materials. The surface area of a protection shelter can be increased under two conditions: 1 ) enough logistics remain to construct the shelter without substantial increases in parasitic mass, and 2) the amount of logistics necessary for the shelter size are not excessive beyond any operational, workload, or deployment time limits. One additional caveat is that this determination must be made considering the conversion of logistics to wastes over the course of the mission to ensure that the shelter can still be created from alternate materials later in the mission. In general, the size and shape of any shelter concept must trade between the desire to increase habitability and work efficiency with more surface area access to subsystems, displays, etc., and the desire reduce the protection area to reduce mass

\section{E. Design of Interior Subsystems and Accommodations for Better Radiation Protection}

Another consideration currently being investigated as part of an effort to further reduce storm shelter masses is the identification of potential subsystem and accommodation design modifications which benefit the overall vehicle design through shelter mass savings. One example under consideration involves utilizing alternate materials for primary and secondary structures, packaging, life support systems, and clothing. Primary and secondary structures currently made of aluminum could potentially be replaced by proven shielding materials such as polyethylene, or by 
novel materials being investigated which explore the possibility of impregnation of materials by hydrogen (e.g., Boron Nitride Nanotubes (BNNT), composites). Packaging for day-to-day items are already made of mostly plastics and foils, but a potential addition is making Cargo Transfer Bags (CTBs) out of higher concentrations of plastics which provide better radiation protection. Life support tanks could be made of polyethylene instead of aluminum, and clothing made of plastic polymers could be selected to contribute as better radiation protection trash. The difficulty with these materials substitutions will be certification for flammability limits and justifying the cost to make modifications to currently adequate systems.

Modifying the shapes of the accommodations themselves to provide better radiation protection is also being worked proactively with subsystem discipline experts, particularly to support continuing work on crew quarters based water wall shelters. One area of particular interest is the reshaping of contingency water tanks to wall-based structures or bags which more evenly distribute a few inches thickness of water across a larger surface area. This causes increases in the mass of the tank hardware, but has a significant probability of reducing total vehicle mass through savings in the radiation protection parasitic mass. Similarly, replacement of existing soft goods water storage bags which are surface area efficient with bags that roll or fold out to provide greater areas of protection can also provide total mass savings. Another concept it to modify the design of solid waste containers similar to those used on ISS to be interlocking bricks to build walls of solid waste protection.

\section{F. Dynamic Logistics Modeling and Strategies to Protect Shelter Materials}

In current radiation shelter design studies, the dynamic changes of habitat interior layout as goods are used and wastes are generated must be investigated to prove that radiation shelters can be fully outfitted at any time during the mission. Figure 9 shows the conversion of logistics into waste streams focusing on those materials good for radiation protection. Because of the consumption of food (which was identified as a promising relocatable logistic for deployable shelters) limits its use throughout the mission for radiation protection, one or more of the waste streams will have to replace the use of food later in the mission. This is challenging because of the requirements limiting biological contamination of the habitat. Two primary options are being investigated in current studies: solid waste and trash.

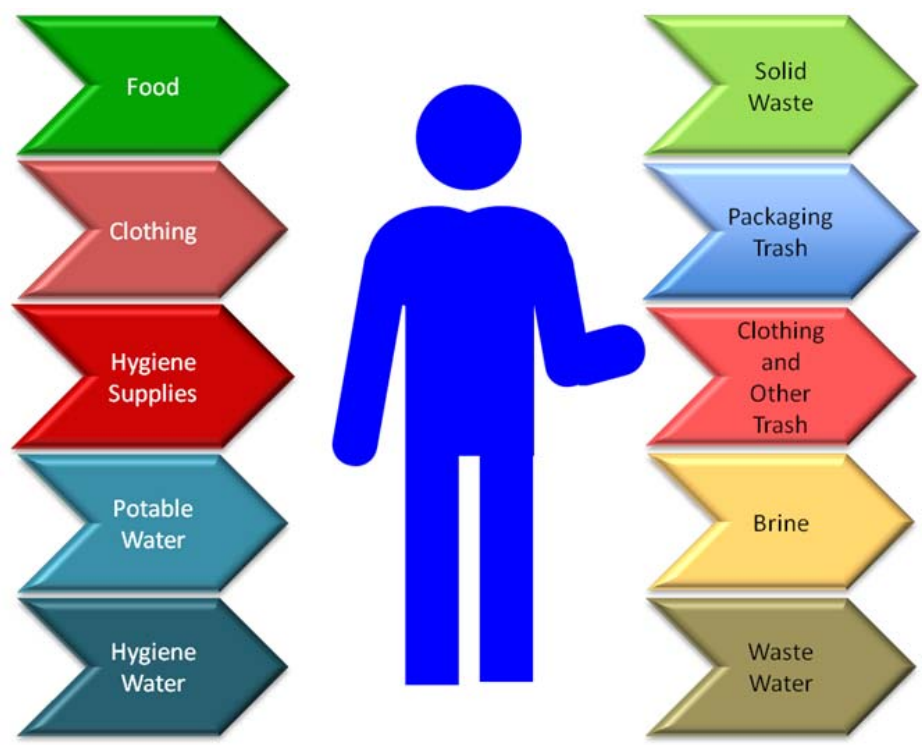

Figure 9: Conversion of logistics to waste streams

Solid waste is nominally stored in either small canisters (e.g. ISS solid waste canisters) or in a waste tank. Modification of these containment units to allow local protection around astronauts requires work to encourage uniform coverage and relocatability. Trash is typically stored for disposal, but several concepts for compaction and safening have been proposed. Current studies are investigating using a heat melt compactor (HMC) to create safened bricks which can be used to replace food pouches in logistics-based protection schemes. Initial studies indicate that the production rate for HMC bricks is approximately one 9 inch square disk per day, which would be insufficient to make up the surface area and thicknesses necessary to sustain a level of protection. In reality, some 
mix of food, HMC bricks, and solid waste would probably be required to ensure robustness throughout a mission and possible off-nominal events.

Materials which are not nominally used during a mission such as contingency water and spares may be chosen because of their independence to mission duration. Another option is to bring more water or operate life support systems at lower levels of closure to yield larger amounts of consumables to increase robustness of the protection concepts to mission durations. This is viable so long as the mass of the integrated vehicle does not increase.

\section{Conclusions and Future Work}

This paper has detailed habitat design considerations and recommendations to improve the integration and performance of radiation shelter concepts into long duration habitat designs. An obvious conclusion from this work is that developing robust and minimal mass SPE radiation protection concepts requires integration of habitability, life support, logistics, and radiation shelter designers early in the design process of habitat concepts. Another observation is that the SPE environment beyond the magnetosphere is substantially more challenging than the SPE environment in LEO, and this may require novel solutions to prevent growth of the vehicle mass which could impact mission feasibility. Currently, the concepts presented in Section II are being matured through continued design iteration and these integration issues identified in Section III are being investigated to optimize the designs. In addition, future work is necessary to understand how interior layouts dynamically change over the mission duration and how to utilize waste streams to counteract this.

Other future work includes investigating the applicability of shelter concepts to shorter duration missions where less logistics and consumables are available for protection materials. Continued maturation of probabilistic SPE models is also needed to determine if the requirements for SPE protection will be as stringent as the Xapsos model, as these exposures significantly affect the design of shelter concepts.

\section{Acknowledgments}

The authors wish to acknowledge the contributions of all of the members of the AES RadWorks Storm Shelter Team:

Thomas L. Jordan (Technical lead), H. Lee Abston, Robert C. Andrews, Heather L. D. Altizer, Sherry B. Araiza, Vincenzo M. Le Boffe, W. David Castle, Jeffrey A. Cerro, Adam M. Gallegos, Kara Latorella, Nicole A. Hintermeister, Samuel James, William M. Langford, Lee Noble, Allison S. Popernack, Edward J. Shea, Judith J. Watson, and Sandy R. Webb.

The authors also wish to recognize the contributions of the AES RadWorks Leadership Team, both past and present, for championing this work and providing valuable guidance:

Bobbie G. Swan (project manager), Deborah M. Tomek (former deputy project manager), M. David Moore, (acting deputy project manager), Catherine D. Mcloed (system engineering and integration lead), E. Neal Zapp (former project scientist), and Edward J. Semones (project scientist).

\section{References}

${ }^{1}$ Walker, S., et al., "Radiation Exposure Analyses Supporting the Development of Solar Particle Event Shielding Technologies”, AIAA- - , AIAA 43 ${ }^{\text {rd }}$ International Conference on Environmental Systems, San Diego, CA, 2013.

${ }^{2}$ NASA, “Habitat Demonstration Unit - Deep Space Habitat”, NASA Fact Sheet, FS-2011-08-047-JSC, 2011.

${ }^{3}$ Toups, L., Simon, M., Smitherman, D., and Spexarth, G., "Design and Parametric Sizing of Deep Space Habitats Supporting NASA’s Human Space Flight Architecture Team,” GLEX-2012.05.3.5x12280, Global Space Exploration Conference, Washington, D.C, 2012.

${ }^{4}$ E. L. Christiansen, K. Nagy, D. M. Lear, T. G. Prior, “Space Station MMOD Shielding,” Acta Astronautica 65, pp 921-929, 2009.

${ }^{5}$ Barghouty, A. F., "Radiation Protection and the iHab”, draft report, August 2010 (unpublished)

${ }^{6}$ Simonsen, L. C., Wilson, J. W., Kim, M. H. and Cucinotta, F. A., "Radiation Exposure for Human Mars Exploration,” Health Physics, Volume 79, Number 5, pp. 515-525, November 2000.

${ }^{7}$ Tim Collins, DSH Super SDM Structures report, Sept. 29, 2011 (unpublished)

${ }^{8}$ Singleterry, R. C., et al., “OLTARIS: On-Line Tool for the Assessment of Radiation in Space,” Acta Astronautica, Vol. 68, 2011, pp. 1086-1097. 
${ }^{9}$ National Council on Radiation Protection and Measurements (NCRP), "Radiation Protection Guidance for Activities in Low-Earth Orbit,” NCRP Report 132, 2000.

${ }^{10}$ Wilson, J. W., et al., "HZETRN: Description of a Free-Space Ion and Nucleon Transport and Shielding Computer Program,” NASA TP-3495, 1995.

${ }^{11}$ Slaba, T. C., Blattnig, S. R., and Badavi, F. F., "Faster and more Accurate Transport Procedures for HZETRN,” Journal of Computational Physics, Vol. 229, 2010, pp. 9397-9417.

${ }^{12}$ Kramer, R., et al., "All about FAX: A Female Adult Voxel Phantom for Monte Carlo Calculations in Radiation Protection Dosimetry,” Physics in Medicine and Biology, Vol. 49, 2004, pp. 5203-5216.

${ }^{13}$ Xapsos, M. A., Barth, J. L., Stassinopoulos, E. G., Burke, E. A., and Gee, G. B., "Space Environmental Effects: Model for Emission of Solar Protons (ESP) - Cumulative and Worst-Case Event Fluences,” NASA TP-1999-209763, 1999.

${ }^{14}$ NASA, "Human Integration Design Handbook”, NASA/SP-2010-3407, 2010.

${ }^{15}$ Tullis, T.and Bied, B., "Space Station Functional Relationships Analysis Final Technical Report, NASA-CR-177497," NASA, 1988. 